\section{Fetal hemoglobin rescues ineffective erythropoiesis in sickle cell disease}

\author{
Sara El Hoss, ${ }^{1,2,3}$ Sylvie Cochet, ${ }^{1,2,3, \#}$ Auria Godard, ${ }^{1,2,3, \#^{\circ}}$ Hongxia Yan, ${ }^{4}$ Michaël \\ Dussiot, ${ }^{5}$ Giacomo Frati, ${ }^{6,7}$ Bénédicte Boutonnat-Faucher, ${ }^{8}$ Sandrine \\ Laurance,,$^{1,2,3}$ Olivier Renaud, ${ }^{9,10,11,12}$ Laure Joseph, ${ }^{13}$ Annarita Miccio, ${ }^{6,7}$ \\ Valentine Brousse, ${ }^{1,2,3,8}$ Narla Mohandas ${ }^{4}$ and Wassim El Nemer ${ }^{1,2,3^{\circ}}$
}

${ }^{1}$ Université de Paris, INSERM UMRS1134, BIGR, Paris, France; ${ }^{2}$ Institut National de la Transfusion Sanguine, Paris, France; ' 2 Laboratoire d'Excellence GR-Ex, Paris, France; ${ }^{4}$ Red Cell Physiology Laboratory, New York Blood Center, New York, NY, USA; ${ }^{5}$ nstitut Imagine, INSERM U1163, CNRS UMR8254, Université de Paris, Hôpital Necker Enfants Malades, Paris, France; 'Laboratory of Chromatin and Gene Regulation during Development, INSERM UMR1163, Paris, France; ' Université de Paris, Imagine Institute, Paris, France; ${ }^{8}$ Service de Pédiatrie Générale et Maladies Infectieuses, Hôpital Universitaire Necker Enfants Malades, Paris, France; 'Institut Curie, Paris Sciences et Lettres Research University, Paris, France; ${ }^{10}$ Institut National de la Santé et de la Recherche Médicale, INSERM U934, Paris, France $;{ }^{11}$ Centre National de la Recherche Scientifique, INSERM UMR3215, Paris, France; ${ }^{12}$ Cell and Tissue Imaging Facility (PICTIBiSA), Institut Curie, Paris, France and ${ }^{13}$ Service de Biothérapie, Hôpital Universitaire Necker Enfants Malades, Paris, France

-Present affiliation: Etablissement Français du Sang PACA-Corse, Aix Marseille Université, EFS, CNRS, ADES, "Biologie des Groupes Sanguins", Marseille, France.

${ }^{\text {"SC }}$ and AG contributed equally as co-second authors.

\section{ABSTRACT}

W hile ineffective erythropoiesis has long been recognized as a key contributor to anemia in thalassemia, its role in anemia of sickle cell disease (SCD) has not been critically explored. Using in vitro and in vivo derived human erythroblasts we assessed the extent of ineffective erythropoiesis in SCD. Modeling the bone marrow hypoxic environment, we found that hypoxia induces death of sickle erythroblasts starting at the polychromatic stage, positively selecting cells with high levels of fetal hemoglobin ( $\mathrm{HbF})$. Cell death was associated with cytoplasmic sequestration of heat shock protein 70 and was rescued by induction of $\mathrm{HbF}$ synthesis. Importantly, we document that in the bone marrow of SCD patients similar cell loss occurs during the final stages of terminal differentiation. Our study provides evidence for ineffective erythropoiesis in SCD and highlights an anti-apoptotic role for $\mathrm{HbF}$ during the terminal stages of erythroid differentiation. These findings imply that the beneficial effect on anemia of increased HbF levels is not only due to the increased life span of red cells but also a consequence of decreased ineffective erythropoiesis.

\section{Introduction}

Sickle cell disease (SCD) is an autosomal hereditary recessive disorder caused by a point mutation in the $\beta$-globin gene resulting in a Glu-to-Val substitution at the sixth position of the $\beta$-globin protein. The resulting abnormal hemoglobin $(\mathrm{HbS})$ polymerizes under hypoxic conditions driving red blood cell (RBC) sickling. ${ }^{1}$ SCD is a multisystem disease characterized by hemolytic anemia, high susceptibility to infections, recurrent painful vaso-occlusive crises, strokes, acute chest syndrome and organ failure.,3

While the pathobiology of circulating RBC has been extensively analyzed in $\mathrm{SCD}$, erythropoiesis is surprisingly poorly documented. In $\beta$-thalassemia, ineffective erythropoiesis is characterized by high levels of apoptotic erythroblasts during the late stages of terminal differentiation, due to an accumulation of free $\alpha$ globin chains. ${ }^{4-6}$ Ineffective erythropoiesis is the major cause of anemia in $\beta$-tha-

\section{Ferrata Storti Foundation}

Haematologica 2021

Volume 106(10):2707-2719

\section{Correspondence: \\ WASSIM EL NEMER \\ wassim.el-nemer@efs.sante.fr}

Received: July 1, 2020

Accepted: August 17, 2020.

Pre-published: August 27, 2020.

https://doi.org/10.3324/haematol.2020.265462

(C)2021 Ferrata Storti Foundation

Material published in Haematologica is covered by copyright. All rights are reserved to the Ferrata Storti Foundation. Use of published material is allowed under the following terms and conditions:

https://creativecommons.org/licenses/by-nc/4.0/legalcode. Copies of published material are allowed for personal or internal use. Sharing published material for non-commercial purposes is subject to the following conditions:

https://creativecommons.org/licenses/by-nc/4.0/legalcode, sect. 3. Reproducing and sharing published material for commercial purposes is not allowed without permission in writing from the publisher. 
lassemia patients. In contrast, a marked decrease in the life span of circulating red cells, a feature of sickle red cells, is considered to be the major determinant of chronic anemia in SCD. It is generally surmised that ineffective erythropoiesis contributes little to anemia. There have been, however, a number of sporadic reports that suggest defective terminal erythroid differentiation in SCD. For example, erythroblasts differentiated in vitro or isolated from bone marrow of SCD patients were shown to sickle under hypoxic conditions.? Such sickling was also reported in the SAD mouse model, with altered morphology of late stage erythroid precursors within the bone marrow, as well as high levels of hemoglobin polymers and increased cell fragmentation occurring during medullary endothelial migration of reticulocytes. ${ }^{8}$ It was presumed that sickling of erythroblasts could lead to ineffective terminal erythroid differentiation. The study of $\mathrm{Wu}$ et al. showed for the first time evidence of ineffective erythropoiesis occurring in the bone marrow of transplanted SCD patients with the preferential survival of the donor erythroid cells in a small cohort of patients. ${ }^{9}$

In the present study, we performed a detailed characterization of terminal erythroid differentiation in non-transplanted SCD patients using both in vivo and in vitro assay systems to critically assess the extent of ineffective erythropoiesis. We documented in both our in vivo and in vitro studies, the occurrence of ineffective erythropoiesis at late stages of terminal erythroid differentiation reflected by high cell death rates between the polychromatic and the orthochromatic stages. We explored the potential mechanistic basis for ineffective erythropoiesis in SCD patients and showed that the molecular mechanism responsible for cell death is likely related to HbS polymerization and its interaction with chaperone protein HSP70 leading to its cytoplasmic sequestration. Importantly, we documented that increased expression of fetal hemoglobin $(\mathrm{HbF})$ can rescue differentiating erythroblasts from cell death.

\section{Methods}

\section{Biological samples}

The study was conducted according to the declaration of Helsinki with approval from the Medical Ethics Committee (GR-Ex/CPP-DC2016-2618/CNILMR01). All SCD patients were of SS genotype. Blood bags from SCD patients enrolled in an exchange transfusion program, bone marrow aspirates from five SCD patients undergoing surgery and bone marrow tissues from five non-anemic donors undergoing hip/sternum surgery, were obtained after informed consent, from Necker-Enfants Malades Hospital (Paris, France) and the North Shore-LIJ Health System (New York, USA) under Institutional Review Board (IRB) approval. Control blood bags from healthy donors were obtained from the Etablissement Français du Sang (EFS).

\section{In vitro differentiation of human erythroid progenitors}

Peripheral blood mononuclear cells were isolated from blood samples after Pancoll fractionation (PAN Biotech). CD34+ cells were then isolated by a magnetic sorting system (Miltenyi Biotec CD34 Progenitor cell isolation kit) following the supplier protocol. $\mathrm{CD} 34^{+}$cells were placed in an in vitro two-phase liquid culture system, as previously described..$^{10}$ For detailed protocols please refer to the Online Supplementary Appendix.

For cultures treated with pomalidomide, cells were incubated with $1 \mu \mathrm{M}$ pomalidomide (Sigma) as previously described, ${ }^{11}$ starting from day 1 (D1) of phase II of culture. For $\gamma$-globin derepression experiments using CRISPR/Cas9, patient $\mathrm{CD} 34^{+}$cells were immunoselected and cultured for 48 hours (h) and then electroporated with ribonucleoprotein (RNP) complexes containing Cas9-GFP protein $(4.5 \mu \mathrm{M})$ and the -197 guide RNA (gRNA) targeting both $H B G 1$ and $H B G 2 \gamma$-globin promoters (5' ATTGAGATAGTGTGGGGAAGGGG 3'; protospacer adjacent motif in bold) or a gRNA targeting the Adeno-associated virus integration site 1 (AAVS1; 5' GGGGCCACTAGGGACAGGATTGG 3'; protospacer adjacent motif in bold). ${ }^{12}$ Cleavage efficiency was evaluated in cells harvested 6 days after electroporation by Sanger sequencing followed by tracking of indels by decomposition (TIDE) analysis. ${ }^{13}$

\section{Imaging flow cytometry analysis of human bone marrow samples}

Bone marrow samples were processed as previously described. ${ }^{14}$ Detailed protocol are stated in the Online Supplemental Appendix.

\section{Flow cytometry}

Cells were analyzed using a BD FACScanto II flow cytometer and BD LSR Fortessa SORP flow cytometer (BD Biosciences) and acquired using the Diva software version 8 (BD Biosciences). Data was analyzed using FCS Express 6 software (DeNovo Software). Detailed protocols of cell staining are stated in the Online Supplemental Appendix.

\section{Cell fractionation and western-blot}

Cytoplasmic and nuclear protein fractions were extracted from erythroblasts at D7 of phase II of culture using the NE-PER nuclear and cytoplasmic kit (Pierce-Thermo Scientific). Ten $\mu g$ of nuclear and cytoplasmic proteins were analyzed by SDS-PAGE, using $10 \%$ polyacrylamide gels, followed by immunoblotting. The antibodies used were rabbit anti-HSP70, mouse anti-actin and mouse anti-lamin $\mathrm{A} / \mathrm{C}$ as a control for the nuclear extract. Proteins were revealed using electrochemiluminescence (ECL) clarity (Biorad) and the Chemidoc MP imaging system (Biorad). Analysis was performed using Image Lab (Biorad). Antibodies details are stated in the Online Supplemental Appendix.

\section{Co-immunoprecipitation assays}

Co-immunoprecipitation of HSP70 and hemoglobin was performed with lysates of 10 million RBC from SCD patients that were either exposed to hypoxia or not for one hour. HSP70 was immunoprecipitated by incubating the lysates with mouse antiHSP70 antibody (Enzo Lifesciences) overnight at $4^{\circ} \mathrm{C}$ followed by a 45 -minute incubation with protein-G sepharose beads (Cytiva-GE-Healthcare) at $4^{\circ} \mathrm{C}$. Eluted proteins were analyzed by SDS-PAGE using a $4-12 \%$ polyacrylamide gel, followed by immunoblotting with mouse anti- $\alpha$-globin (Santa Cruz Biotechnology) or rabbit anti-HSP70 (SANTA-CRUZ) antibodies. Proteins were revealed using ECL clarity and the Chemidoc MP imaging system.

\section{Confocal microscopy and proximity ligation assay}

Co-immunolocalization and proximity ligation assays (PLA) were performed with cells from D7 of phase II of culture. Acquisition was made on LSM700 Zeiss confocal microscope using Zen software. Analysis was performed using Fiji. ${ }^{15}$ Detailed protocols are stated in the Online Supplemental Appendix.

\section{Statistical analysis}

Statistical analyses were performed with GraphPad Prism 
(version 7). The data was analyzed using Mann-Whitney unpaired test and Wilcoxon paired test, as indicated in the figure legends.

\section{Results}

\section{Hypoxia-induced cell death during in vitro terminal erythroid differentiation}

The bone marrow environment has been well documented to be hypoxic $\left(0.1-6 \% \mathrm{O}_{2}\right)$. $^{16-18}$ As hypoxia induces $\mathrm{HbS}$ polymerization, we hypothesized that cell death may occur in vivo because of HbS polymer formation in the late stages of differentiation characterized by high intracellular hemoglobin concentration. In order to test our hypothesis, we performed in vitro erythroid differentiation using $\mathrm{CD}^{+} 4^{+}$cells isolated from SCD patients and from healthy donors. A two-phase erythroid differentiation protocol was used and cultures were performed at two different oxygen conditions, i.e., normoxia and partial hypoxia $\left(5 \% \mathrm{O}_{2}\right)$, starting at D3 of the second phase, at which time hemoglobin synthesis begins to increase markedly (Online Supplementary Figure S1). The choice of $5 \% \mathrm{O}$, was made since it falls at the high end of the reported oxygen tension range of the hematopoietic niche $\left(0.1-6 \% \mathrm{O}_{2}\right){ }^{17}$ and because it drives $\mathrm{HbS}$ polymerization and cell sickling, as we previously reported. ${ }^{19}$ First, we performed video microscopy experiments with nucleated SCD erythroblasts at D9 of culture and confirmed their ability to sickle at $5 \% \mathrm{O}_{2}$ (Figure 1A; Online Supplementary Video S1). Differentiation of control erythroblasts showed no difference in the general waterfall pattern between normoxia and hypoxia (Figure 1B), although hypoxia translated into a consistently higher but not statistically significant increase in total cell count (Figure 1C). Under normoxia, SCD differentiation showed a mild deceleration till D9 as compared to control (Figure 1B), with a proliferation that was negatively impacted by hypoxia (Figure 1C). Under both oxygen conditions, cell proliferation was significantly higher in the control than in the SCD cultures, starting from D7 (Figure 1C). May Grünwald-Giemsa (MGG) staining confirmed the differentiation delay of SCD cells, especially under hypoxia where cells seemed to accumulate at the polychromatic stage when compared to control cells (Figure 1D). In addition, higher proportions of enucleated cells were found in control cells at D9 (Figure 1D) and D11 (Figure 1E). Enucleation was improved under hypoxia for control erythroblasts while it was significantly diminished for SCD cells (Figure 1E), indicating a negative impact of hypoxia at this critical maturation step in the context of SCD.

In order to assess if the decrease in proliferation in SCD was due to cell death, we measured the percentage of apoptotic cells in the cultures by staining the glycophorin A (GPA)-positive cells with annexin V (Figure 1F). The percentage of annexin $\mathrm{V}^{+}$cells was higher in SCD than in control cultures under both oxygen conditions at D7 and D9, with a greater variability among SCD than in control cells (Figure 1G). Furthermore, the extent of apoptosis of SCD cells was higher under hypoxia than under normoxia at both time points while no difference was noted for control cells (Figure 1G). Altogether, these findings imply that even under a conservative choice of $5 \% \mathrm{O}$, to mimic hypoxia in bone mar- row there is increased cell death during the terminal differentiation stages in SCD cells only.

\section{F-cells are enriched during sickle cell disease erythroid differentiation}

Using flow cytometry, we measured the percentage of cells expressing $\mathrm{HbF}$ (F-cells) during in vitro differentiation (Figure 2A). The percentage of F-cells (\%F-cells) in control cultures fell within the reported range of $20-40 \%,{ }^{20,21}$ while it was very variable for SCD cells reaching more than $70 \%$ at D9 (Figure 2B). Interestingly, there was no difference of $\%$ F-cells between normoxia and hypoxia for control cells, while for SCD, \%F-cells was higher under hypoxia than under normoxia for all of the six independent primary cell samples (Figure 2B). Taken together with the apoptosis data, these findings imply that F-cells were positively selected under hypoxia in SCD. This inference was supported by flow cytometry data showing higher percentages of dead cells, based on the fixable viability stain (FVS), within the non-F-cell population as compared to the F-cell population for SCD cells (Figure 2C and D). In contrast, these percentages were similar between both cell populations in the control (Figure $2 \mathrm{C}$ and $\mathrm{D}$ ), confirming preferential apoptosis of the cells with low/no HbF expression in the SCD context only.

\section{HSP70 is sequestered in the cytoplasm of non-F-cells}

As cytoplasmic sequestration of the chaperone protein HSP70 by $\alpha$-globin aggregates is associated with cell death during erythropoiesis in $\beta$-thalassemia major, ${ }^{5}$ we investigated if apoptosis of SCD erythroblasts might be due to cytoplasmic trapping of HSP70 by HbS polymers. We performed western blot analyses to quantify HSP70 in the cytoplasmic and nuclear extracts of erythroblasts at D7 of phase II of culture (Figure 3A). There was less HSP70 in the nucleus and more in the cytoplasm of SCD erythroblasts under hypoxia compared to normoxia (Figure 3A), indicating mislocalization of HSP70 in SCD cells under hypoxia. Importantly, these lower amounts of nuclear HSP70 were associated with lower amounts of GATA-1 (Figure 3A), suggesting that cell death under these conditions was likely due to altered protection of GATA-1 by HSP70.

The cells used in these assays were pools of live and apoptotic, F-cells and non-F-cells. In order to better address HSP70 localization in each of these subpopulations, we performed imaging flow cytometry experiments with cells stained for multiple markers. Only Hoechst-positive cells with high GPA expression were taken into consideration to exclude both reticulocytes and proerythroblasts. HSP70 fluorescence intensity was measured in the cytoplasm and the nucleus of live (FVS) and dead (FVS') cells at D7. HSP70 nuclear intensity was lower in dead than FVS cells, for both control and SCD cells under hypoxia (Figure 3B). Moreover, SCD FVS ${ }^{+}$cells showed higher HSP70 cytoplasmic levels than FVS cells, while no difference was detected between both subpopulations for control cells (Figure 3B). These results suggest that hypoxia-induced cell death in the SCD context is likely due to HSP70 entrapment in the cytoplasm. Next, we measured HSP70 intensity in F-cells and non-F-cells. Under hypoxia at D7, HSP70 nuclear intensity was higher in SCD F-cells than non-F cells, while there was no difference between both cell types in control (Figure 3C; Online Supplementary Figure S2A). We then measured the nucleus/cytoplasm (N/C) ratio of HSP70 intensity in polychromatic and orthochromatic cells. The 
cell subtypes were identified based on morphological parameters of nuclear and cytoplasmic areas ${ }^{22}$ (Online Supplementary Figure S2B; Online Supplementary Table S1) that discriminate between the basophilic, polychromatic and orthochromatic erythroblasts (Figure 3D; Online Supplementary Figure S2C and D). HSP70 N/C ratio was significantly higher in SCD F than non-F-cells (Figure 3E) which was not the case for control cells (Online Supplementary Figure S2E), indicating that increased HSP70 cytoplasmic entrapment is a feature of SCD non-F-cells. Although there was no significant difference of HSP70 N/C ratio between SCD F-cells and control, this ratio showed a broader distribution in SCD F-cells (Online Supplementary Figure S2E). We hypothesized that this heterogeneity might be linked to the variable intracellular levels of $\mathrm{HbF}$ in these cells. Using an analysis mask that quantifies the amount of $\mathrm{HbF}$ per cell, we classified F-cells as either low or high F-expressing cells, depending on the intracellular expression level of $\mathrm{HbF}$ (Figure 3F). We found that HSP70 N/C ratio was higher in high F-cells compared to low Fcells (Figure 3G), implying that the amounts of HSP70 trapped in the cytoplasm were inversely related to the cytoplasmic content of $\mathrm{HbF}$ and suggesting that high amounts of $\mathrm{HbF}$ protect $\mathrm{SCD}$ erythroblasts against apopto-
A

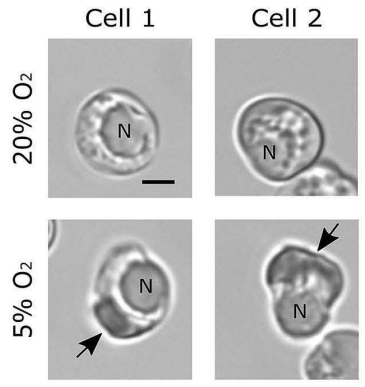

$\mathrm{CN}$

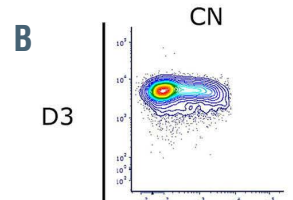

D5

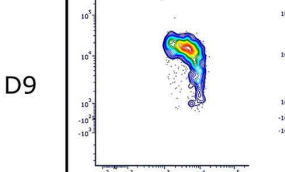

D11

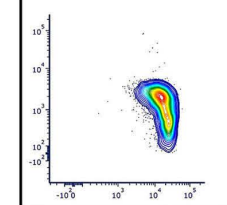

$\mathrm{CH}$
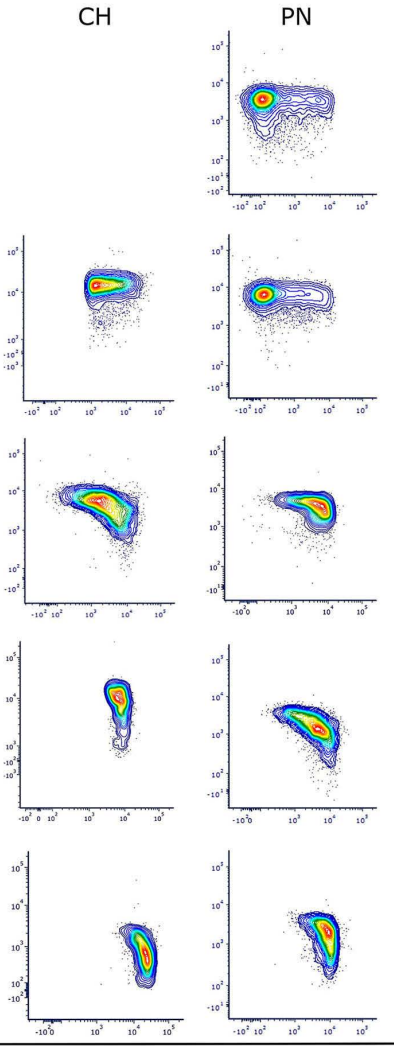

$\mathrm{PH}$
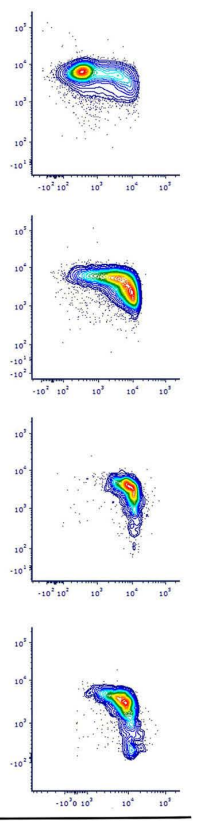

Band 3

D
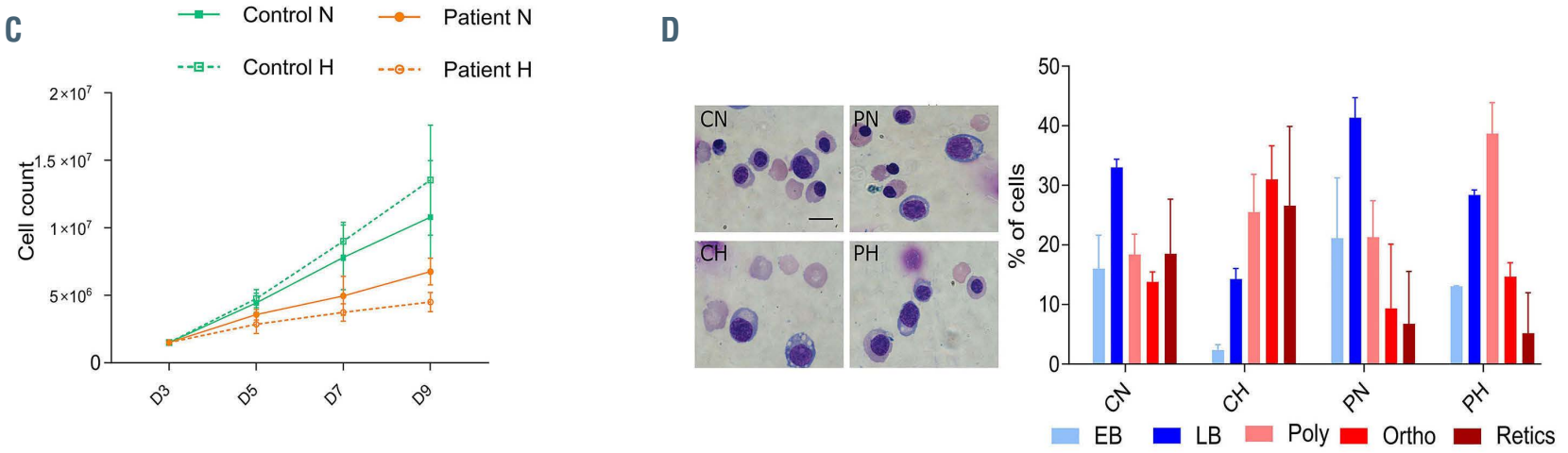

Figure 1. Cell proliferation and apoptosis during terminal erythroid differentiation in vitro under normoxia and partial hypoxia. (A) Microscopy images of two sickle cell disease (SCD) in vitro cultured erythroblasts incubated for 30 minutes at $20 \%$ (upper panel) and $5 \%$ (lower panel) oxygen. "N" represents the nucleus and the arrow points to the $\mathrm{HbS}$ polymers formed under $5 \%$ oxygen; scale bar: $5 \mu \mathrm{m}$. (B) A contour plot representing the distribution of glycophorin A (GPA)-positive cells with respect to the expression of Band 3 (x-axis) and CD49d (y-axis) at day (D) 3, D5, D7, D9 and D11 of phase II of culture in control erythroid precursors under normoxia $(\mathrm{CN})$ or hypoxia $(\mathrm{CH})$, and in patient erythroid precursors under normoxia (PN) or hypoxia (PH). (C) Cell count of erythroid precursors at $D 3,5,7$ and 9 in control $(n=4)$ and patient $(n=6)$ under normoxia $(N)$ and hypoxia $(H)$ (means \pm standard error of the mean [SEM]). (D) May Grunwald-Giemsa staining of erythroid precursors at D9 of phase II of culture (left panel; scale bar: $10 \mu \mathrm{m}$ ) and graph representing the cellular distribution as means \pm SEM of early basophilic (EB), late basophilic (LB), polychromatic (Poly), orthochromatic (Ortho) and reticulocytes (Retics) at D9 of culture of control normoxia (CN), control hypoxia (CH), patient normoxia (PN) and patient hypoxia $(\mathrm{PH})$. (continued on next page) 
E

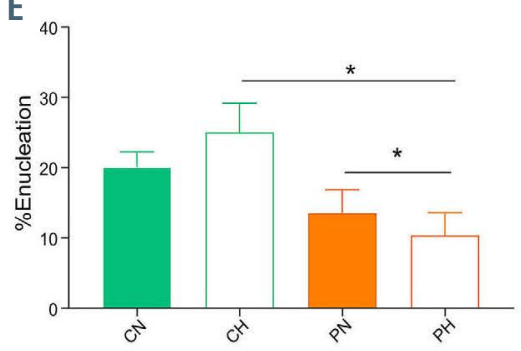

F
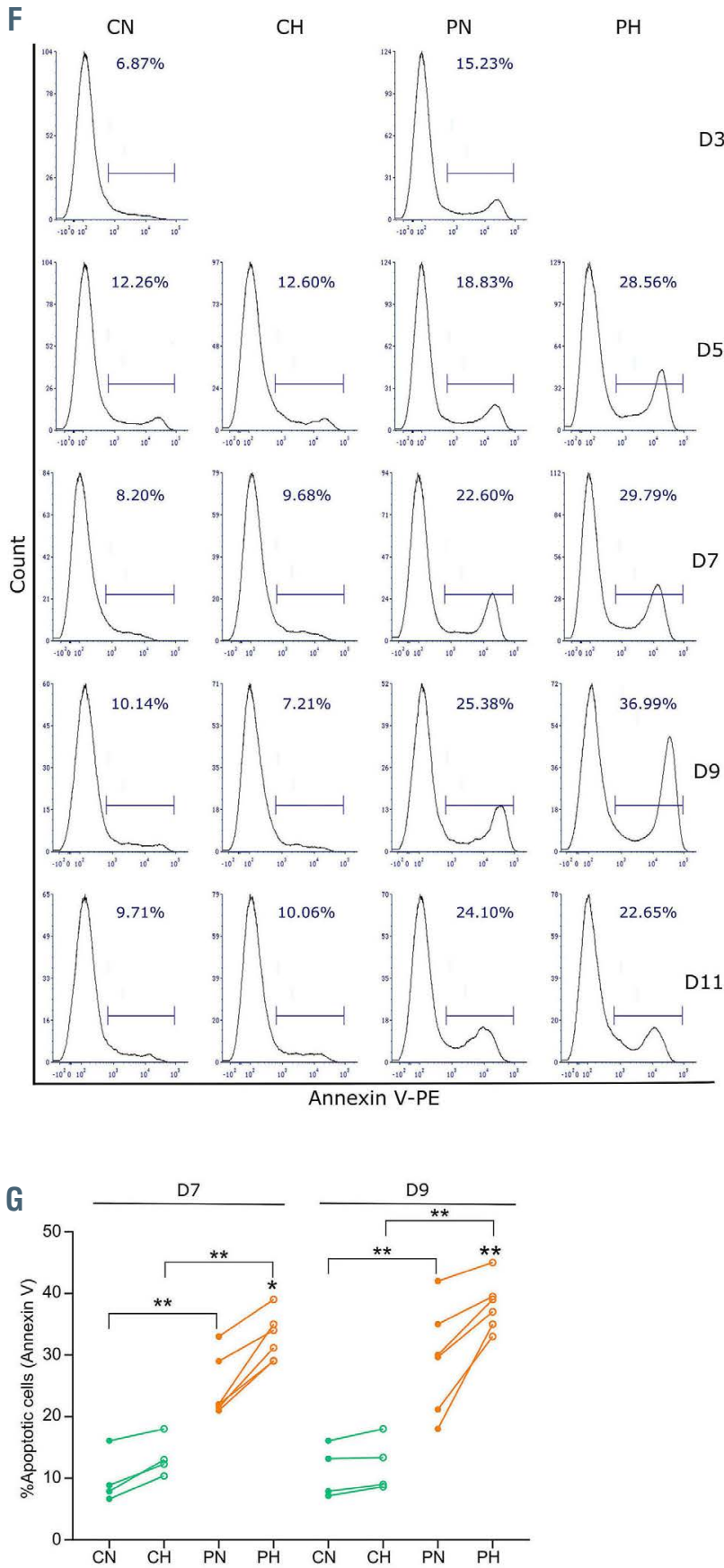

Figure 1. (continued from previous page) (E) Percentage of enucleation measured at $\mathrm{D} 11$ for $\mathrm{CN}, \mathrm{CH}, \mathrm{PN}$ and $\mathrm{PH}$ erythroblasts (means $\pm \mathrm{SEM}$; $\mathrm{CN}$ and $\mathrm{CH}$ : $n=3$; $P N$ and $P H: n=6$ ). (F) Flow cytometry plots showing percentage of apoptotic cells (Annexin V-positive cells) measured at D3, D5, D7, D9 and D11. (G) Percentage of apoptotic cells in control $(n=4)$ and patients $(n=6)$ under normoxia $(\mathrm{N})$ and hypoxia $(\mathrm{H})$ at D7 and D9 of phase II of culture. $* P<0.05, * * P<0.01$ Wilcoxon paired test and Mann-Whitney test (E and $G$ ). sis. This inference was supported by our findings showing lower percentages of low F-cells in SCD cultures as compared to control, as well as lower percentages of SCD low F-cells under hypoxic than normoxic conditions (Figure $3 \mathrm{H})$. On the other hand, the percentage of high F-cells was significantly higher in SCD erythroblasts as compared to control, with even higher percentages under hypoxia (Figure 3I). Of note, no difference in the percentage of low or high F-cells was observed between hypoxia and normoxia for control cells (Figure $3 \mathrm{H}$ and I), indicating that the differences observed for SCD cells are the result of differential cell death related to $\mathrm{HbF}$ expression levels.

\section{HbS-HSP70 protein complex in sickle cell disease cells under hypoxia}

In order to explore the molecular mechanism of cytoplasmic trapping of HSP70, we performed immunofluorescence assays using confocal microscopy and found colocalization of HSP70 and hemoglobin in both control and SCD cells (Figure 4A). We used Pearson's correlation coefficient ${ }^{23,24}$ to assess the degree of co-localization and found no difference between both cell types (data not shown), probably because of the high abundance of hemoglobin in the cytoplasm. In order to overcome this limitation, we performed PLA, that show fluorescent spots when two proteins are at a distance $<40 \mathrm{~nm} .{ }^{25}$ We detected SCD cells with fluorescent spots $\left(\mathrm{PLA}^{+}\right)$under both normoxic and hypoxic conditions (Figure 4B) that were further quantified by imaging flow cytometry (Figure $4 \mathrm{C}$ ). The percentage of $\mathrm{PLA}^{+}$cells was very low in control, with values close to background levels (1-1.5\%) under both normoxia and hypoxia, while SCD cells showed higher values under normoxia $(4.5-18.5 \%)$ with a systematic and significant increase under hypoxia (9.5-36\%) (Figure 4D), indicating proximity between HSP70 and HbS but not HSP70 and $\mathrm{HbA}$. Moreover, PLA+ SCD cells showed higher mean fluorescence intensity under hypoxia than under normoxia (Figure 4D), indicating that hypoxia induces the formation of more potent $\mathrm{HbS}-\mathrm{HSP} 70$ complexes that could account for cytoplasmic retention of HSP70.

In order to explore the potential of $\mathrm{HbS}$ polymers and HSP70 interacting within the same protein complex, we performed co-immunoprecipitation assays. SCD RBC suspensions were placed at normoxia or hypoxia then lysed, and HSP70 was immunoprecipitated. Using an anti- $\alpha$-globin antibody we found co-immunoprecipitation of HSP70 and $\alpha$-globin under hypoxia but not under normoxia (Figure 4E) supporting the presence of HbS-HSP70 protein complex. Of note, despite using the same amounts of RBC for both conditions, there was less immunoprecipitated HSP70 under hypoxia than normoxia likely due to decreased solubility of $\mathrm{HbS}$ polymer fibers under hypoxia as evidenced by the color of the lysates under both conditions and the presence of a small red colored precipitate under hypoxia (Online Supplementary Figure S3).

\section{Cell death during the terminal stages of erythroid differentiation in bone marrow of sickle cell disease patients}

In order to validate the direct relevance of the in vitro findings to in vivo conditions, we analyzed terminal erythroid differentiation using unmanipulated marrow samples from SCD patients. Bone marrow aspirates were obtained from five SCD patients and cells were stained for surface markers GPA, CD49d and Band 3 and analyzed by 
flow cytometry. Differentiating erythroblasts were determined using the CD49d and Band 3 staining pattern within the $\mathrm{GPA}^{+}$population (Figure $5 \mathrm{~A}$ ), as previously described. ${ }^{14}$ Imaging flow cytometry was used to confirm gating of all nucleated cells and cell homogeneity within each of the four gated populations by cellular features of size, morphology and nuclear size and polarization (Figure 5B). We quantified the cells at the early basophilic (EB), late basophilic (LB), polychromatic and orthochromatic stages. Considering the GPA positive population as $100 \%$, the mean percentages of $\mathrm{EB}, \mathrm{LB}$, polychromatic and orthochromatic cells were $5.1 \%, 10.2 \%, 20.4 \%$ and $25.2 \%$ (Figure 5C), indicating loss of the expected cell doubling between the polychromatic and orthochromatic stages (1.24+/-0.1, $P<0.01$; Figure 5D). These results implied that cell death occurs between the polychromatic and orthochromatic stages in a significant proportion of erythroblasts, in accordance with our in vitro data. Similar analysis was performed with bone marrow aspirates of five controls (3\% EB, $6.8 \%$ LB, $13 \%$ polychromatic cells and $24.6 \%$ orthochromatic cells, Figure 5C) that confirmed the expected doubling of cells with successive cell divisions without cell loss between development stages during normal erythropoiesis (Figure 5D). Next, we stained the cells with an anti-HbF antibody to measure the percentage of F-cells at the different stages. There was a significant increase in \%F-cells between the polychromatic $(16.4 \% \pm 4)$ and orthochromatic stages $(32.4 \% \pm 4.79)$ (Figure $5 \mathrm{E}$ and F), concomitant with the cell loss observed between these stages (Figure 5C and
D), indicating preferential survival of F-cells during late stages of erythroblast maturation in vivo and supporting our hypothesis for an anti-apoptotic role of $\mathrm{HbF}$ during in vivo erythropoiesis in SCD.

\section{Induction of fetal hemoglobin protects against cell death}

In order to confirm the anti-apoptotic role of $\mathrm{HbF}$ in SCD erythroblasts we induced its expression in vitro using pomalidomide (POM), an immunomodulatory drug previously shown to induce $\mathrm{HbF}$ expression during erythropoiesis, ${ }^{11,26}$ and determined if higher $\mathrm{HbF}$ levels could rescue the cells from apoptosis. As we were interested in monitoring the stages during which hemoglobin is synthesized, POM was added at D1 of phase II of culture. As expected, POMtreated SCD cultures showed higher percentages of F-cells than untreated cultures (Figure 6A; Online Supplementary Figure $\mathrm{S} 4 \mathrm{~A}) \mathrm{H}$. HbF induction by POM was associated with significantly lower levels of apoptosis compared to untreated cultures under both hypoxia and normoxia (Figure 6B; Online Supplementary Figure S4B). Importantly, there was no significant difference in the apoptosis levels between normoxic and hypoxic conditions of POM-treated cells indicating that the higher F-cell levels protected SCD cells from apoptosis under hypoxia.

In order to specifically address the role of $\mathrm{HbF}$, we used a CRISPR-Cas9 approach that we have recently developed to mimic the effect of hereditary persistence of fetal hemoglobin ( $\mathrm{HPFH}$ ) by disrupting the binding site for $\mathrm{HbF}$
A

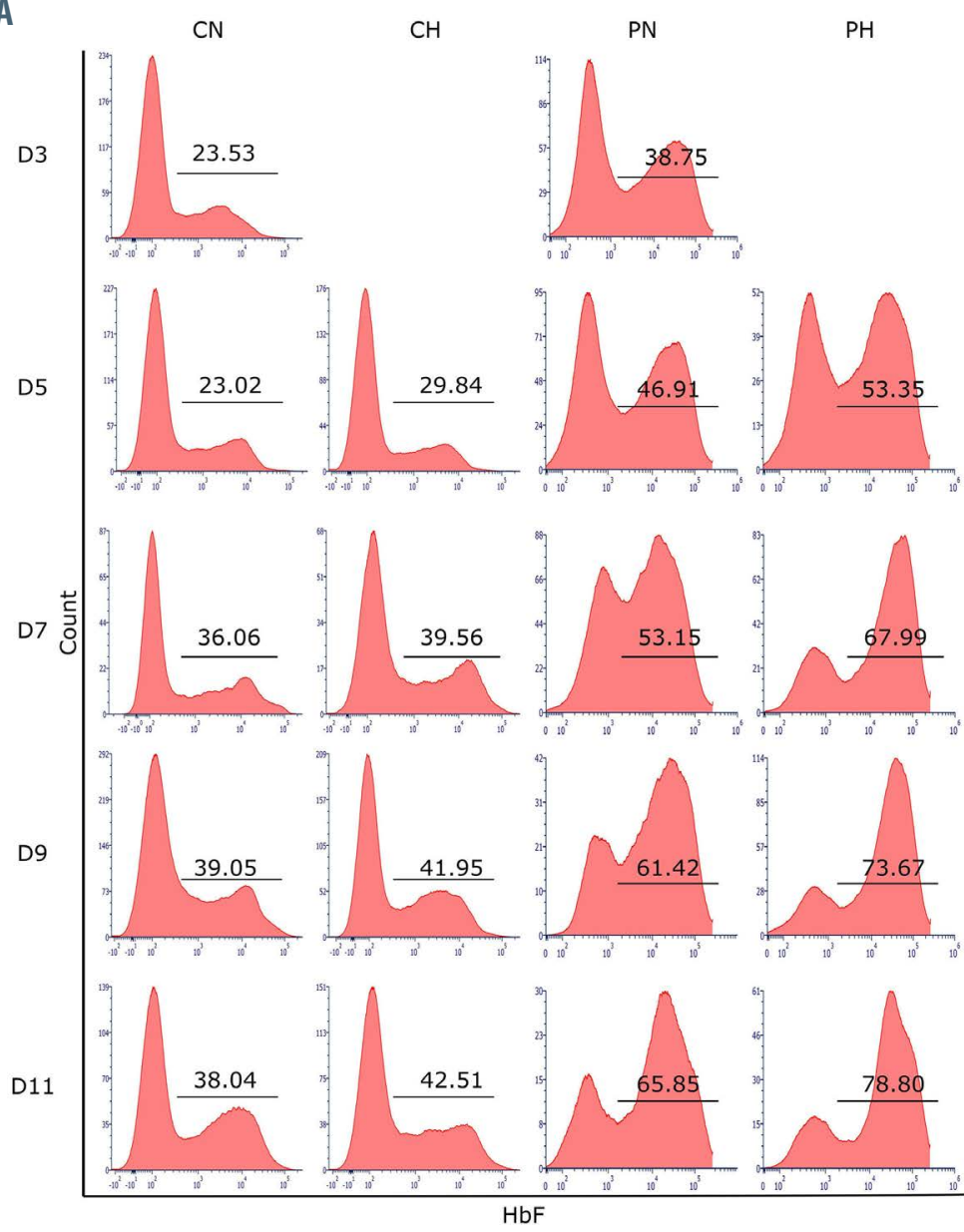

B

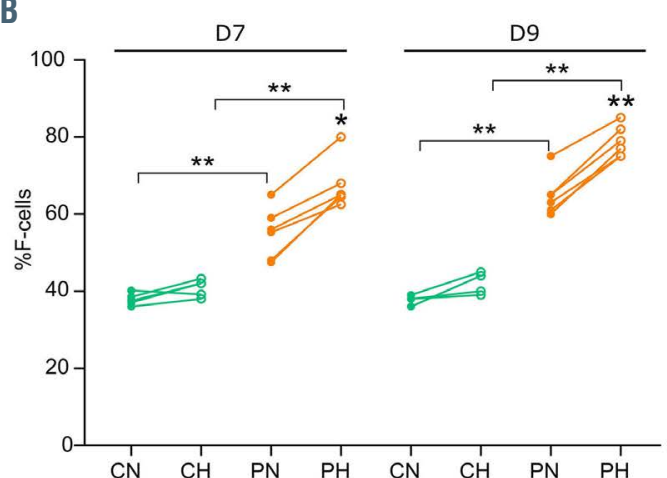

Figure 2. Distribution of erythroid precursors expressing fetal hemoglobin in vitro. (A) Flow cytometry plots showing the percentage of cells expressing fetal hemoglobin (F-cells) at day (D) 3, D5, D7, D9 and D11 of phase II of culture in control erythroid precursors under normoxia $(\mathrm{CN})$ or hypoxia $(\mathrm{CH})$, and in patient erythroid precursors under normoxia (PN) or hypoxia (PH). (B) Flow cytometry plots showing dead cells, measured by fixable viability stain (FVS APC-Cy7), in non-F-cells (F-) (blue curve) and F-cells ( $\mathrm{F}+$ ) (red curve) of control $(\mathrm{CH})$ and patient $(\mathrm{PH})$ cells under hypoxia. (C) Percentage of F-cells measured at D7 and D9 of phase II of culture in control $(n=4)$ and patient cells $(n=6)$ under normoxia $(\mathrm{N})$ and hypoxia $(\mathrm{H})$. (continued on next page). 
C
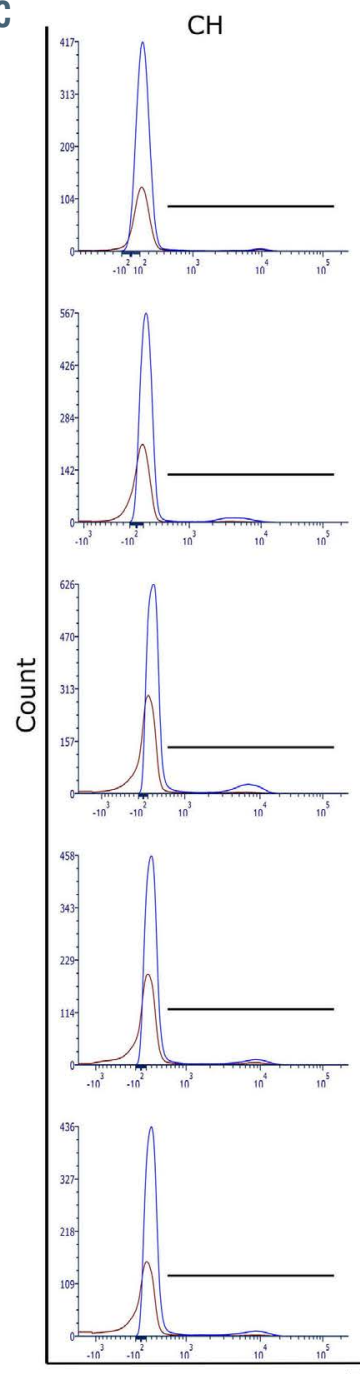

$\mathrm{PH}$
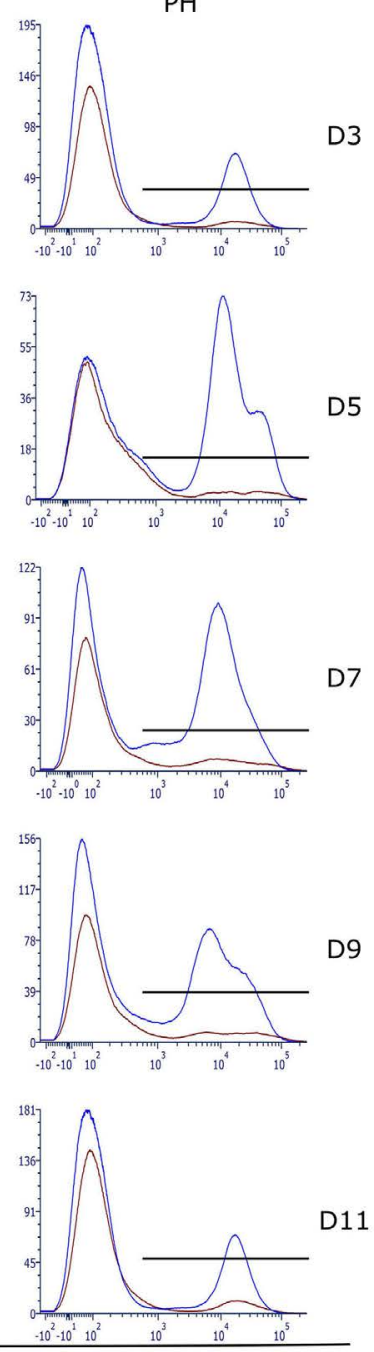

FVS APC-Cy7 repressor LRF in the fetal $\gamma$-globin promoters (HBG1 and $H B G 2)^{12}$ (Online Supplementary Figure S4C). CD34 cells were transfected either with a gRNA targeting the LRF binding site (-197) or a gRNA targeting an unrelated locus (AAVS1). Genome editing efficiency at the $\gamma$-globin promoter ranged between $23.2 \%$ and $73.5 \%$, as determined by Sanger sequencing at D6 of phase I of culture. Cells were grown and differentiated under normoxia or hypoxia. As expected, the disruption of the LRF binding site resulted in $\mathrm{HbF}$ induction as shown by higher \%F-cells compared to AAVS1 control (Figure 6C; Online Supplementary Figure S4D). These higher levels of F-cells resulted in decreased apoptosis, under both normoxic and hypoxic conditions (Figure 6D; Online Supplementary Figure S4E), clearly demonstrating the positive and selective effect of $\mathrm{HbF}$ on SCD cell survival.

\section{Discussion}

Ineffective erythropoiesis has been previously suggested to be a feature of $\mathrm{SCD}^{79,27}$ but it has not been critically evaluated and documented. Our present findings provide direct evidence for ineffective erythropoiesis in SCD patients, with significant cell death occurring at the late

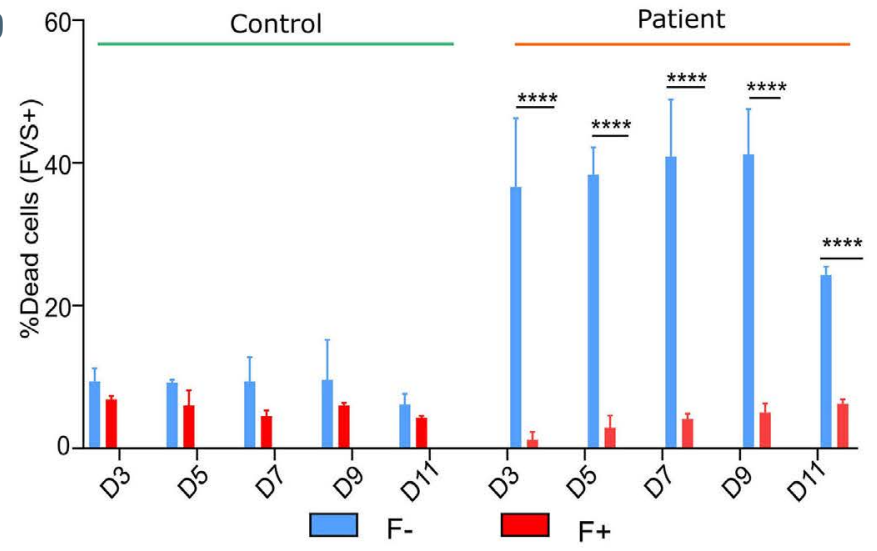

Figure 2. (continued from previous page). (D) Percentage of dead cells (means \pm standard error of the mean) measured in the F- and F+ subpopulations for control and patient cells under hypoxia. ${ }^{*} P<0.05, * * P<0.01, * * * * P<0.0001$; Wilcoxon paired test and Mann-Whitney test (C); Mann-Whitney test (D)

stages of terminal erythroid differentiation in vivo.

Among previous reports, the study by $\mathrm{Wu}$ and collaborators has highlighted abnormalities during erythropoiesis in the bone marrow of transplanted SCD patients by showing progressive intramedullary loss of SCD erythroblasts and relative enrichment of donor erythroid precursors at the onset of hemoglobinization in a small cohort of patients. ${ }^{9}$ Our results further demonstrate significant cell death between the polychromatic and the orthochromatic stages, when cellular HbS concentration reaches sufficiently high levels to promote polymer formation under partial hypoxia. Our in vivo data unequivocally document the occurrence of ineffective erythropoiesis in non-transplanted SCD patients, in the absence of confounding factors like conditioning drugs and exogenous donor-related factors that can impact the hematopoietic niche and interfere with the survival of patient's erythroblasts.

This study also reveals a new role for HbF in SCD by showing that it protects a subpopulation of differentiating erythroblasts from apoptosis, both in vivo and in vitro. $\mathrm{HbF}$ is a known modulator of disease severity in SCD as it inhibits $\mathrm{HbS}$ polymerization at the molecular level, preventing or attenuating RBC sickling and alleviating disease complications. ${ }^{28,29}$ In healthy adults, $\mathrm{HbF}$ accounts 
A

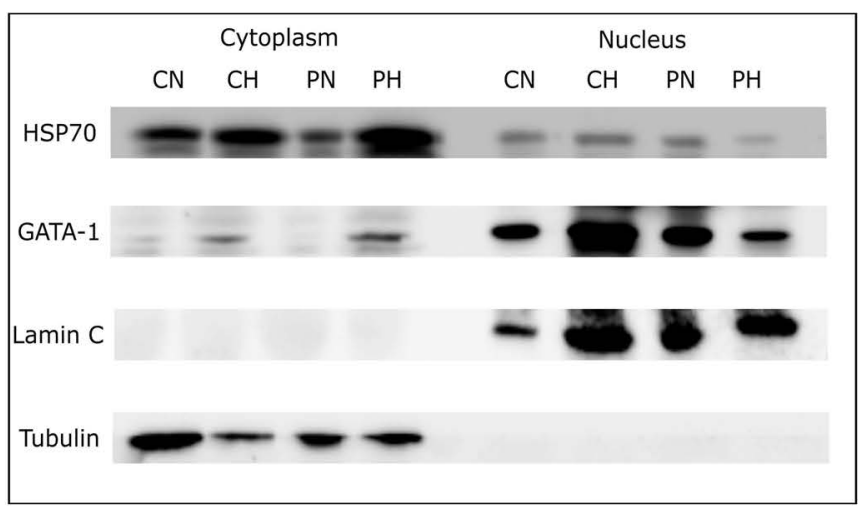

B
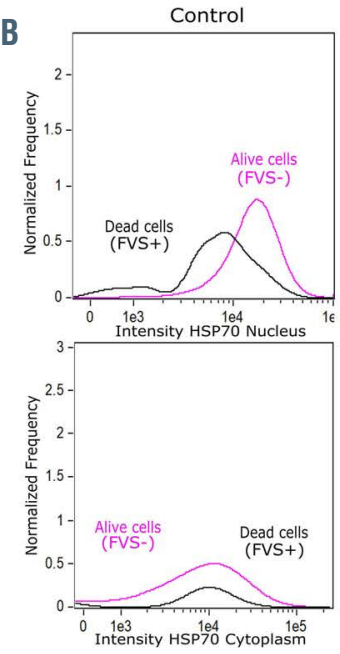
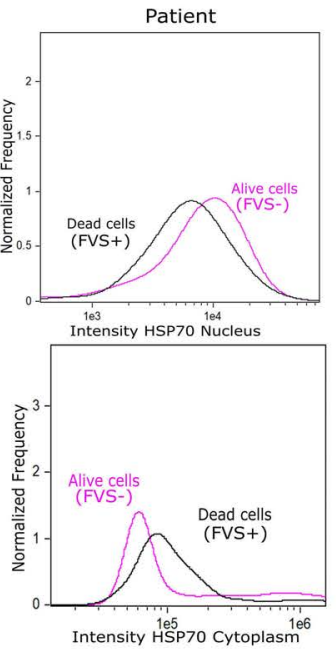

C
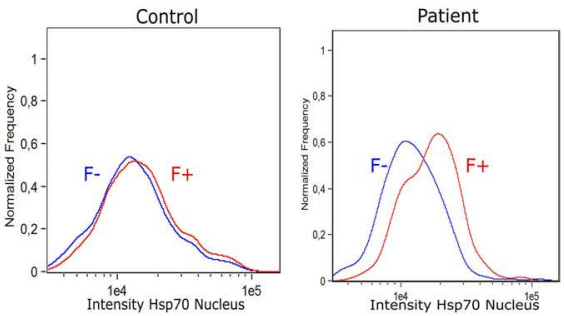

E

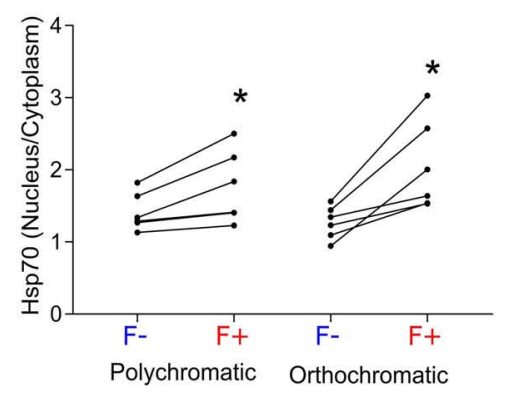

D

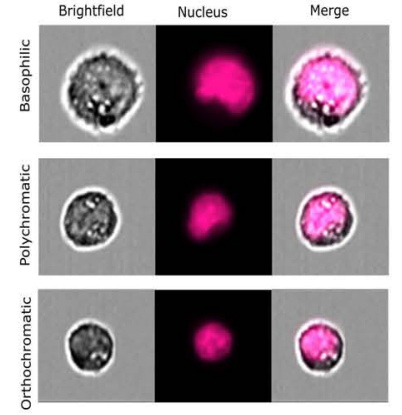

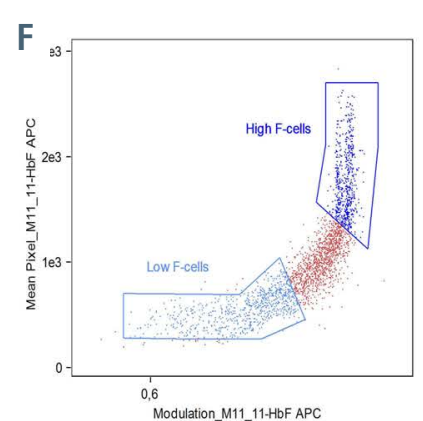

G

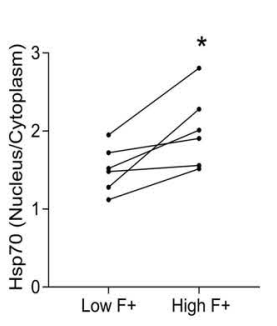

H

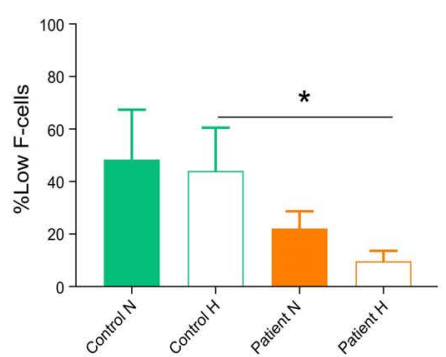

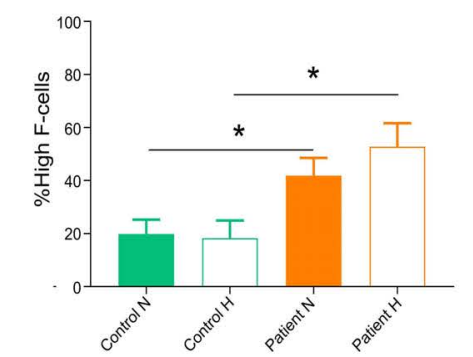

Figure 3. HSP70 cytoplasmic and nuclear distribution. (A) Western blot analysis of HSP70, GATA-1, lamin C and tubulin performed on cytoplasmic and nuclear extracts of erythroid precursors at day 7 (D7) of phase II of culture from control normoxia (CN), control hypoxia (CH), patient normoxia (PN) and patient hypoxia (PH). (B) Distribution of the nuclear intensity (upper panel) and cytoplasmic intensity (lower panel) of HSP70 at D7 of phase II of culture in dead cells (FVS+) and live cells (FVS-) of control and patient cells under hypoxia. (C) Distribution of the nuclear intensity of HSP70 at D7 of phase II of culture in the F- and F+ subpopulations of control (left) and patient (right) cells under hypoxia. (D) Images of basophilic, polychromatic and orthochromatic subpopulations obtained by imaging flow cytometry. (E) HSP70 nucleus/cytoplasm ratio in F- and F+ polychromatic and orthochromatic patient erythroblasts at D7 of phase II of culture ( $n=6$ ). (F) Dot plot representing the modulation mask of fetal hemoglobin (HbF) (x-axis) and mean pixel HbF values (y-axis) used to discriminate between low and high F-cells. (G) HSP70 nucleus/cytoplasm ratio in low and high F-cells of patients' orthochromatic erythroblasts at D7 of phase II of culture under hypoxia ( $n=6)$. Percentage of (H) Low $F$ cells and (I) High F-cells in control $(n=3)$ and patient $(n=6)$ cells at normoxia and hypoxia at D7 of phase II of culture (means \pm standard error of the mean). $* P<0.05$. Wilcoxon paired test $(\mathrm{E}$ and $\mathrm{G})$ and Mann-Whitney unpaired test ( $\mathrm{H}$ and $\mathrm{I})$. FVS: fixable viability stain; F-cells: cells expressing fetal hemoglobin. 
for less than $1 \%$ of total hemoglobin ${ }^{30}$ and is restricted to a small subset of RBC (2\%) called F-cells. ${ }^{31-33}$ In SCD, the expression of $\mathrm{HbF}$ is higher than in healthy individuals and varies considerably among patients. Although the mechanisms underlying increased expression of $\mathrm{HbF}$ are not completely elucidated, stress erythropoiesis and preferential survival of F-cells in the circulation are suggested contributing factors. ${ }^{34-38}$ Our findings show that high $\mathrm{HbF}$ levels not only increase survival of circulating red cells but also play a role in the preferential survival of erythroblasts under physiologically relevant hypoxic conditions in the bone marrow.
A
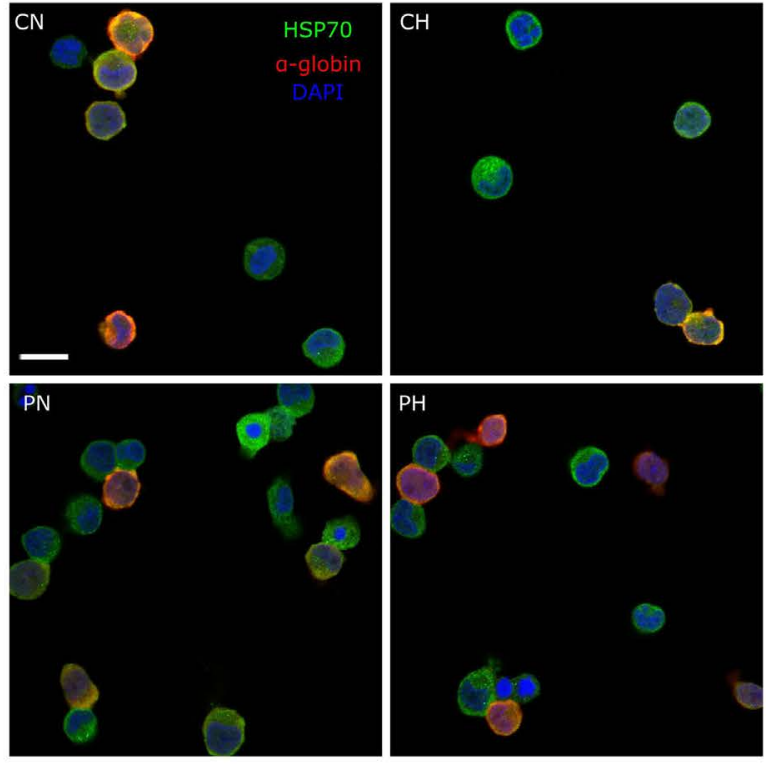

B
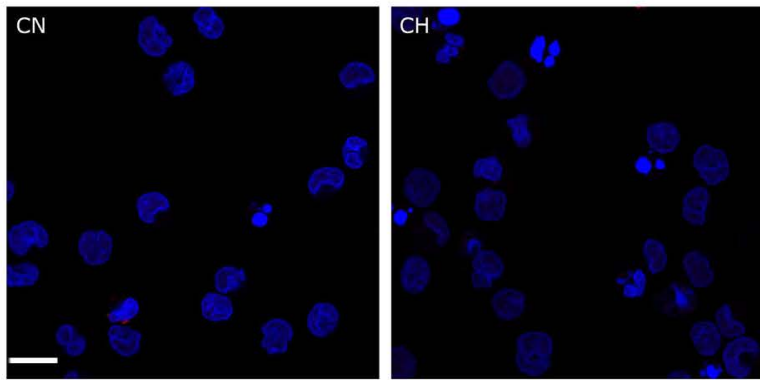

PN

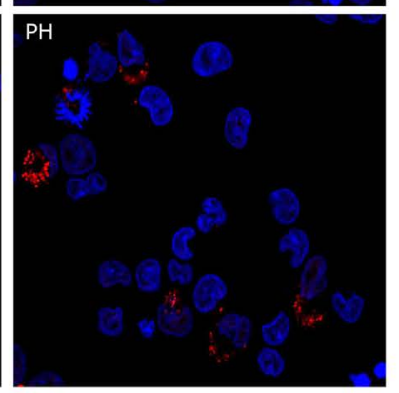

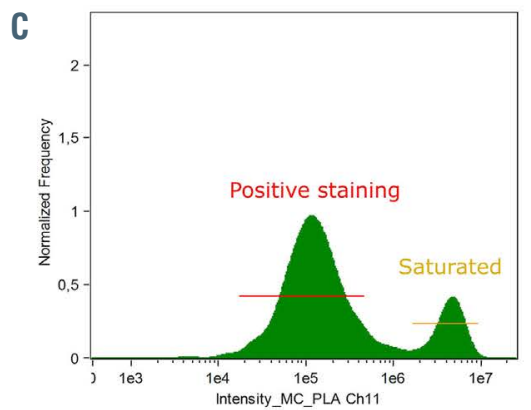
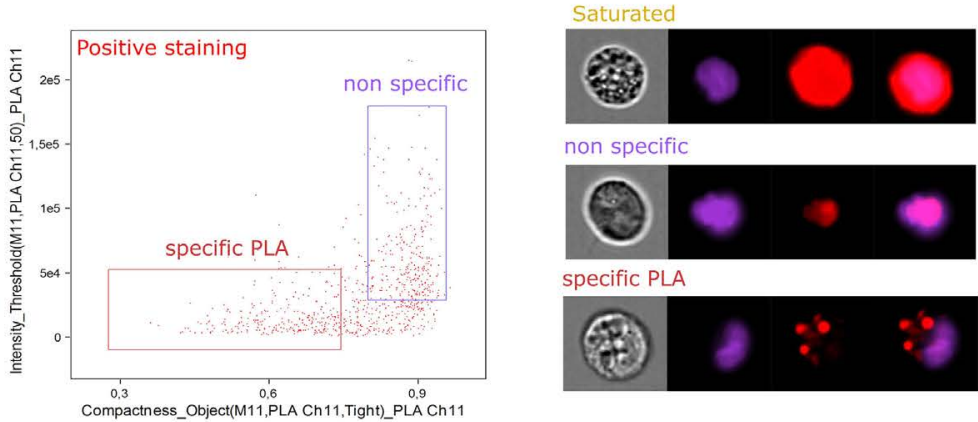

non specific

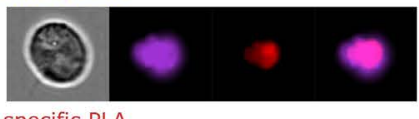

specific PLA

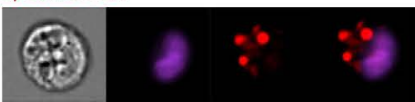

D

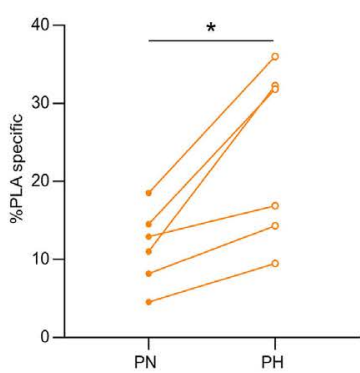

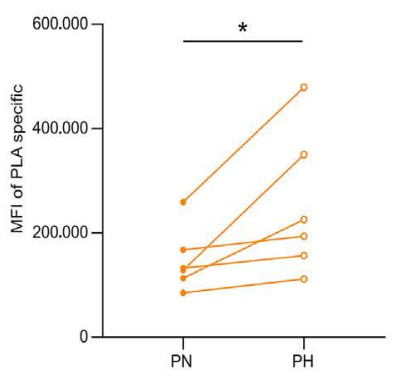

$\mathbf{E}$

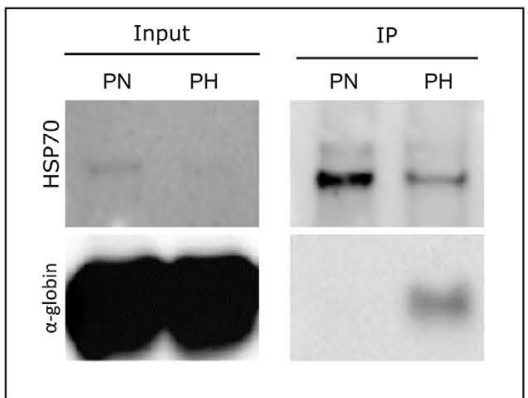

Figure 4. HSP70 and $\alpha$-globin colocalization and co-immunoprecipitation. (A) Confocal microscopy images of control erythroid precursors under normoxia (CN) or hypoxia (CH), and of patient erythroid precursors under normoxia (PN) or hypoxia (PH) at day 7 (D7) of phase II of culture showing colocalization (in yellow) of HSP70 (green) and $\alpha$-globin (red); nucleus is in blue $(n=3)$; scale bar: $10 \mu \mathrm{m}$. (B) Proximity ligation assay (PLA) between HSP70 and $\alpha$-globin at D7 of phase II of culture. Red spots indicate proximity $(<40 \mathrm{~nm})$ between both proteins. Spots were observed in PN and PH cultures, while no spots were seen in $\mathrm{CN}$ and $\mathrm{CH}$. A representative image of each culture is shown ( $n=3)$; scale bar: $10 \mu \mathrm{m}$. (C) (Left) A histogram representing the intensity of APC signal generated by PLA, a gating of positive staining and saturated staining is indicated. (Middle) A dot plot representing an analysis mask using the compactness feature (x-axis) and intensity feature (y-axis) to discriminate between PLA-specific staining and non-specific staining. (Right) Representative images of each gate. (D) Percentage of cells (left) and mean fluorescence intensity (right) of PLA-specific staining in erythroid precursors of patient normoxia (PN) and patient hypoxia (PH) at D9 of phase II of culture ( $\mathrm{n}=6)$. (E) Co-immunoprecipitation assay of HSP70 with $\alpha$-globin using circulating sickle cell disease (SCD) red blood cells incubated under normoxia (PN) or hypoxia (PH) for 1 hour. HSP70 and $\alpha$ globin bands are detected in the lysates (left panel) and after HSP70 immunoprecipitation (right panel). ${ }^{*} P<0.05$, Wilcoxon paired test (D). 
Our findings show that in vitro induction of $\mathrm{HbF}$ by pomalidomide rescues SCD erythroblasts from cell death and improves their differentiation, in accordance with improved efficiency of erythropoiesis in vivo in treated SCD mice. ${ }^{39}$ Furthermore, results using genetically modified $\mathrm{CD} 34^{+}$cells strongly imply that overexpression of $\mathrm{HbF}$ and concomitant down-regulation of $\mathrm{HbS}^{12}$ in a specific and selective manner corrects the apoptosis observed during erythroid differentiation, which is therefore presumably a feature of $\mathrm{HbS}$.
HSP70 is a chaperone protein that plays an important role during erythropoiesis by protecting GATA-1 from cleavage by caspase- 3 in the presence of Epo, ${ }^{40}$ thus promoting normal terminal differentiation. From a mechanistic perspective, sequestration of HSP70 in the cytoplasm through interaction with $\mathrm{HbS}$ polymers is therefore a possible mechanism for ineffective erythropoiesis in SCD. Co-immunoprecipitation of HSP70 and HbS under hypoxia is indeed highly suggestive of such interactions between these proteins. Furthermore, specific HSP70
A

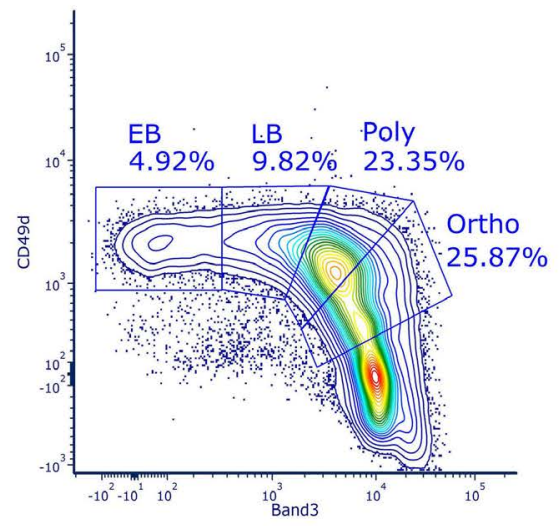

C.

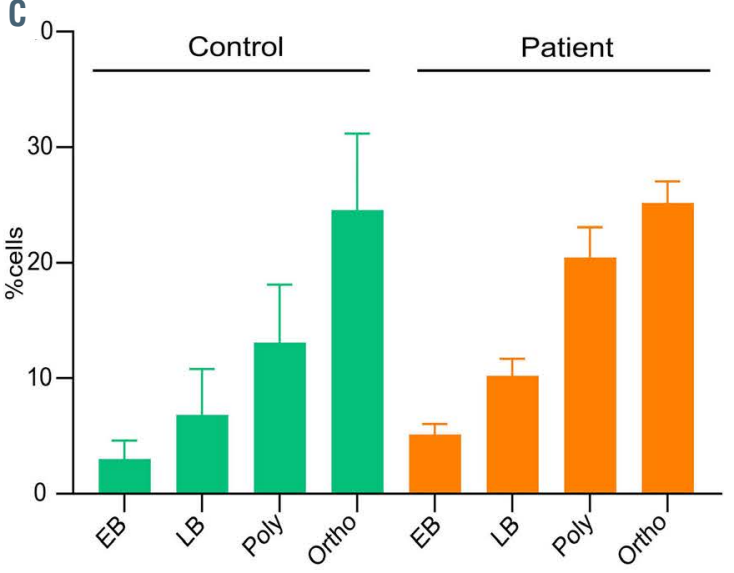

$\mathbf{E}$

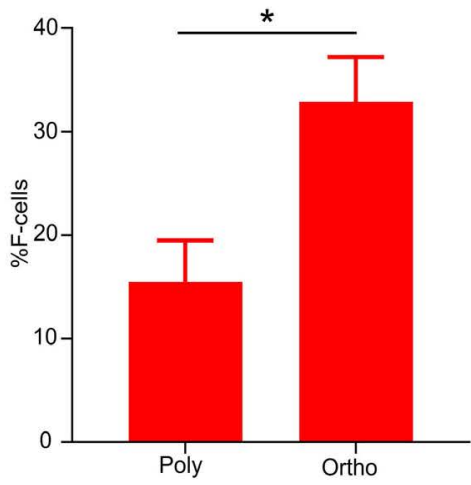

B

Poly

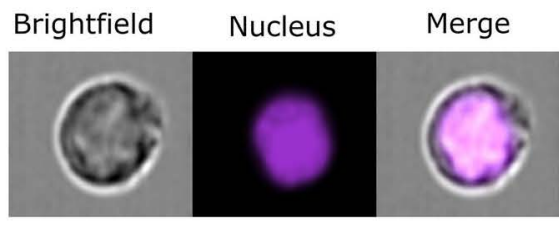

Ortho

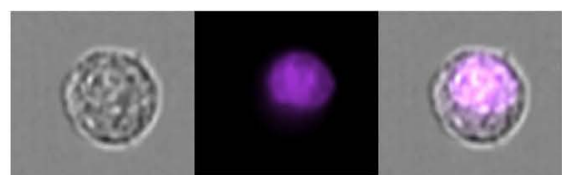

D

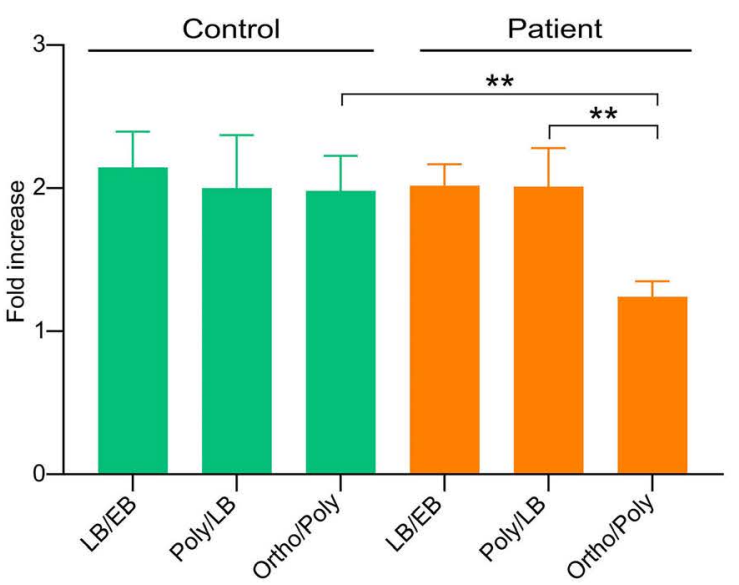

$\mathbf{F}$

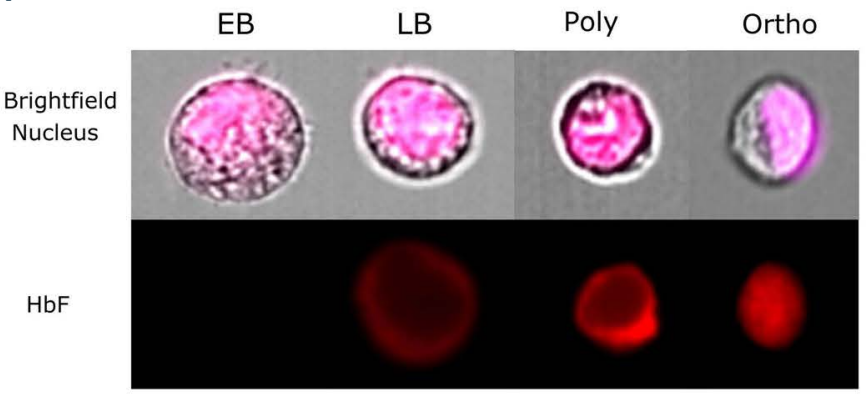

Figure 5. Analysis of human terminal erythroid differentiation in vivo. (A) A contour plot representing the distribution of glycophorin A (GPA)-positive cells with respect to Band 3 (x-axis) and CD49d (y-axis) from a bone marrow sample of a sickle cell disease (SCD) patient. (B) Images obtained using imaging flow cytometry from the gating of polychromatic (Poly) and orthochromatic (Ortho) erythroblasts. Nucleus was stained with Hoechst. (C) Percentage of cells at the early basophilic (EB), late basophilic (LB), polychromatic (Poly) and orthochromatic (Ortho) stages in five bone marrow samples of controls (green) and SCD patients (orange) (means \pm standard error of the mean [SEM]). (D) The fold increase of cells between the EB and LB (LB/EB), LB and Poly (Poly/LB), Poly and Ortho (Ortho/Poly) stages in five controls and five SCD patients (means \pm SEM). (E) Percentage of cells expressing fetal hemoglobin (F-cells) in vivo in the polychromatic (Poly) and orthochromatic (Ortho) subpopulations of the patient bone marrow samples $(n=5)$ (means \pm SEM). (F) Imaging flow cytometry images of early basophilic (EB), late basophilic (LB), polychromatic (Poly) and orthochromatic (Ortho) precursors. Upper images are a merge of brightfield and nucleus, lower images are for fetal hemoglobin (HbF) staining (red). $\star P<0.05, * * P<0.01$; Mann-Whitney test ( $\mathrm{D}$ and $\mathrm{E})$. 
sequestration in the pool of SCD dead cells under hypoxia could account for the lower HSP70 nuclear content of these cells and the subsequent lower GATA-1 nuclear levels. As the molecular event that initiates HSP70 trapping in SCD, namely $\mathrm{HbS}$ polymerization, occurs in cells with high cellular concentration of $\mathrm{HbS}$, required for polymer formation, our results show the absence of cell death in the early stages of differentiation where the intracellular concentration of $\mathrm{HbS}$ is likely insufficient to induce sickling. Likewise, low levels of $\mathrm{HbF}$ are less protective against cell death, since a minimal threshold of intracellular $\mathrm{HbF}$ is needed for a protective polymer-inhibiting effect. ${ }^{41}$ At physiological levels of hypoxia, there is however not an exclusive selection of F-cells, as significant amounts of cells with no/low $\mathrm{HbF}$ were found to complete erythroid differentiation. Further studies are needed to fully address the biological mechanisms underlying this observation and the commitment of erythroid progenitors to the F lineage in SCD.
Finally, this study sheds light on the importance of applying partial hypoxia during erythroid differentiation in vitro in order to mimic in vivo conditions in $\mathrm{SCD}$. In our view, this together with cell proliferation and apoptosis are important parameters to consider in assessing the beneficial impact of therapeutic approaches in SCD such as $\mathrm{HbF}$ induction, ${ }^{10,20,42}$ anti-sickling molecules such as voxelotor ${ }^{43,44}$ or gene therapy aiming at expressing a therapeutic $\beta$-globin. ${ }^{45}$ In addition, specific targeting of ineffective erythropoiesis should presumably have a major beneficial clinical impact.

In summary, our study shows that $\mathrm{HbF}$ has a dual beneficial effect in SCD by conferring a preferential survival of F-cells in the circulation and by decreasing ineffective erythropoiesis. These findings thus bring new insights into the role of $\mathrm{HbF}$ in modulating clinical severity of anemia in SCD by both regulating red cell production and red cell destruction.
A

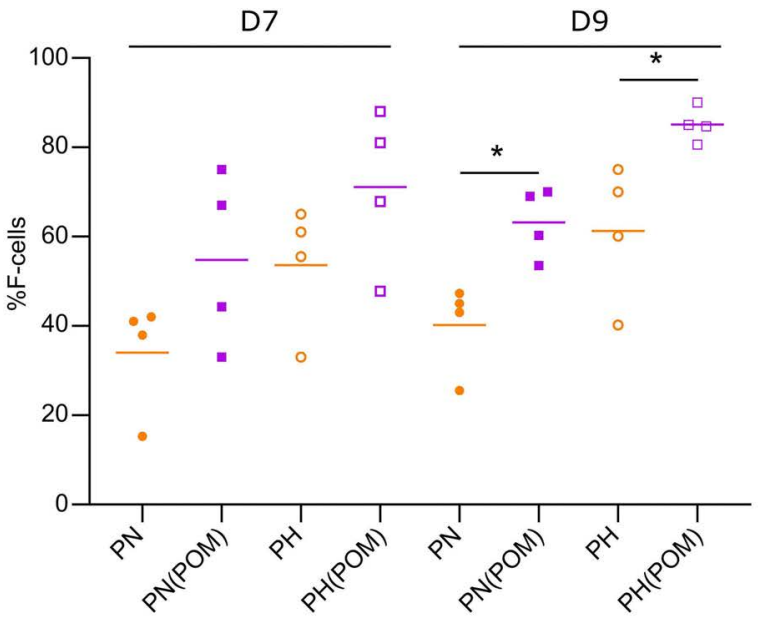

C

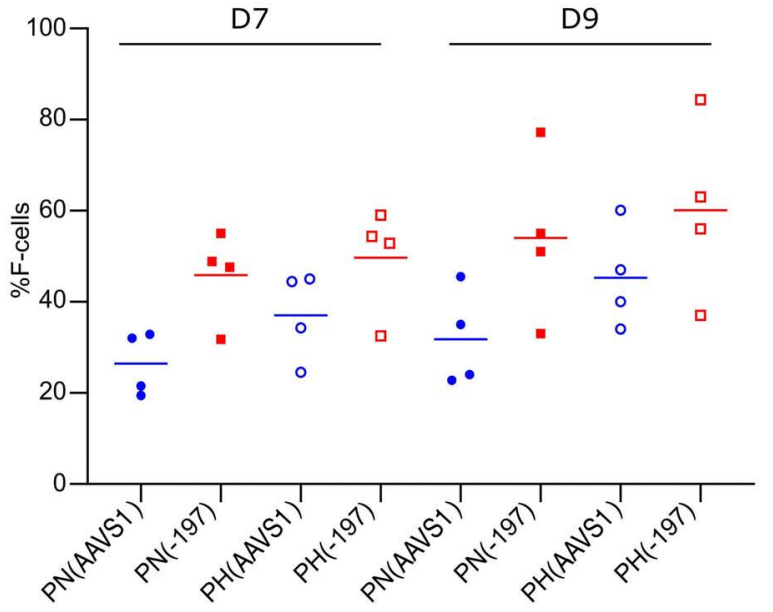

B

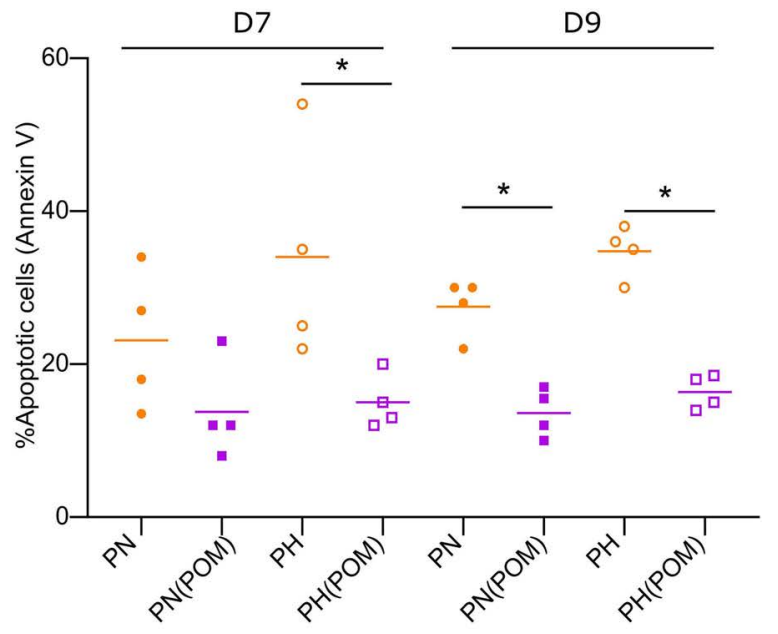

D7

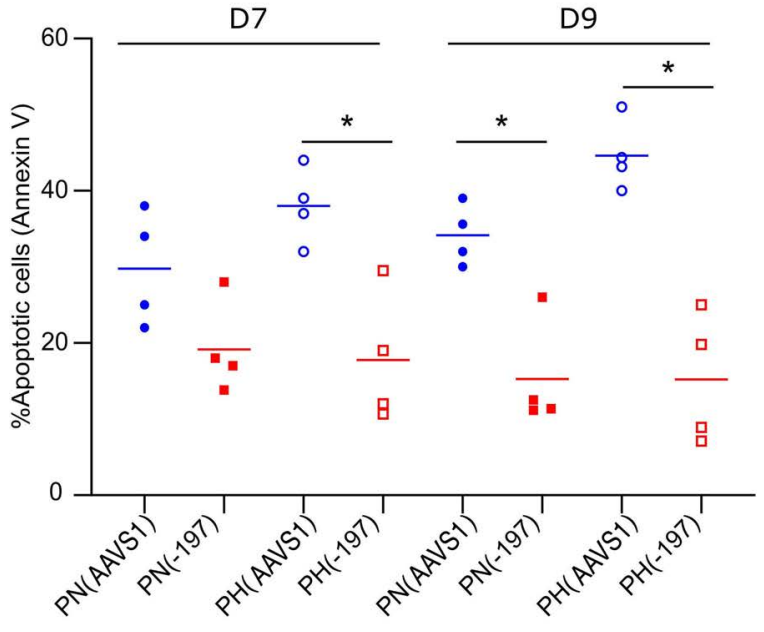

Figure 6. Effect of fetal hemoglobin induction by pomalidomide or CRISPR/Cas9 on terminal erythroid differentiation of sickle cell disease erythroblasts. (A) Percentage of cells expressing fetal hemoglobin (F-cells) at day (D) 7 and D9 of phase II of culture of culture in patient erythroblasts under normoxia (PN), hypoxia $(\mathrm{PH})$, normoxia with POM [PN(POM)] and hypoxia with POM $[\mathrm{PH}(\mathrm{POM})](\mathrm{n}=4)$. (B) Percentage of F-cells at D7 and D9 of phase II of culture in patient erythroblasts under normoxia (PN) and hypoxia (PH) treated with guide RNA (gRNA) targeting the LRF binding site (-197) or an unrelated locus as control (AAVS1) ( $\mathrm{n}=4)$. Genome editing efficiency was $56.1 \pm 9.6 \%$ and $79.2 \pm 2.8 \%$ for -197 and AAVS1 samples, respectively. (C) Percentage of apoptotic cells measured by flow cytometry in PN, $\mathrm{PH}, \mathrm{PN}(\mathrm{POM})$ and $\mathrm{PH}(\mathrm{POM})$ at D7 and D9 of phase II of culture of culture $(\mathrm{n}=4)$. (D) Percentage of apoptotic cells at D7 and D9 of phase II of culture in patient erythroblasts under normoxia (PN) and hypoxia (PH) treated with gRNA targeting the LRF binding site $(-197)$ or an unrelated locus as control (AAVS1) ( $n=4)$. Horizontal bars represent the mean of each group; ${ }^{*} P<0.05$, Mann-Whitney test $(A, B$ and $D)$. 


\section{Disclosures}

No conflicts of interest to disclose.

\section{Contributions}

SEH designed and conducted experiments, acquired and analyzed data and wrote the manuscript; SC, $A G, H Y, M D, G F$ and $S L$ conducted experiments, acquired and analyzed data; $B B F$ and $L J$ provided biological samples; $O R$ and $A M$ supervised experiments and analyzed data; $V B$ provided biological samples and discussed data; NM discussed data and wrote the manuscript, WEN designed research, analyzed data and wrote the manuscript. All authors read and edited the manuscript.

\section{Acknowledgments}

We thank the patients and their families for accepting to be part of this study. We thank Dr. Jean-Philippe Semblat, Dr. Maria Alejandra Lizarralde-Iragorri and Ms Sandrine Genetet for technical support, Dr. Flavia Guillem, Dr. Thiago Trovati Maciel and Dr. Olivier Hermine for helpful discussions, Dr.
Slimane Allali and the nursing staff of Hôpital de jour Pédiatrie Générale of Hôpital Necker Enfants Malades for patient management. We thank Dr. Lionel Blanc for providing non-anemic bone marrow samples.

\section{Funding}

The work was supported by the Institut National de la Santé et de la Recherche Médicale (INSERM), Institut National de la Transfusion Sanguine, and grants from Laboratory of Excellence GR-Ex, reference ANR-11-LABX-0051, EUR G.E.N.E., reference ANR-17-EURE-0013, and NIH grant DK32094. The labex GR-Ex is funded by the IdEx program "Investissements d'avenir" of the French National Research Agency, reference ANR-18-IDEX-0001. PICT-IBiSA is part of the FranceBioImaging infrastructure funded by ANR-10-INBS-04. SEH was funded by the Ministère de l'Enseignement Supérieur et de la Recherche (Ecole Doctorale BioSPC) and received financial support from, addmedica, the Club du Globule Rouge et du Fer and the Société Française d'Hématologie.

\section{References}

1. Pauling L, Itano HA, Singer SJ, Wells IC. Sickle cell anemia, a molecular disease. Science. 1949;110(3):543-548.

2. Piel FB, Steinberg MH, Rees DC. Sickle cell disease. N Engl J Med. 2017;376(16):15611573.

3. Ware RE, de Montalembert M, Tshilolo L, Abboud MR. Sickle cell disease. Lancet. 2017;390(10091):311-323.

4. Arlet JB, Dussiot M, Moura IC, Hermine O, Courtois G. Novel players in beta-thalassemia dyserythropoiesis and new therapeutic strategies. Curr Opin Hematol. 2016;23(3):181-188.

5. Arlet JB, Ribeil JA, Guillem F, et al. HSP70 sequestration by free alpha-globin promotes ineffective erythropoiesis in beta-thalassaemia. Nature. 2014;514(7521):242-246.

6. Rivella S. Ineffective erythropoiesis and thalassemias. Curr Opin Hematol. 2009;16(3): 187-194

7. Hasegawa S, Rodgers GP, Dwyer N, et al. Sickling of nucleated erythroid precursors from patients with sickle cell anemia. Exp Hematol. 1998;26(4):314-319.

8. Blouin MJ, De Paepe ME, Trudel M. Altered hematopoiesis in murine sickle cell disease. Blood. 1999;94(4):1451-1459.

9. Wu CJ, Krishnamurti L, Kutok JL, et al. Evidence for ineffective erythropoiesis in severe sickle cell disease. Blood. 2005;106 (10):3639-3645.

10. McArthur JG, Svenstrup N, Chen C, et al. A novel, highly potent and selective phosphodiesterase- 9 inhibitor for the treatment of sickle cell disease. Haematologica. 2020;105(3):623-631.

11. Dulmovits BM, Appiah-Kubi AO, Papoin J, et al. Pomalidomide reverses gamma-globin silencing through the transcriptional reprogramming of adult hematopoietic progenitors. Blood. 2016;127(11):1481-1492.

12. Weber L, Frati G, Felix T, et al. Editing a gamma-globin repressor binding site restores fetal hemoglobin synthesis and corrects the sickle cell disease phenotype. Sci Adv. 2020;6(7):eaay9392.

13. Brinkman EK, Chen T, Amendola M, van Steensel B. Easy quantitative assessment of genome editing by sequence trace decompo- sition. Nucleic Acids Res. 2014;42(22):e168.

14. Hu J, Liu J, Xue F, et al. Isolation and functional characterization of human erythroblasts at distinct stages: implications for understanding of normal and disordered erythropoiesis in vivo. Blood. 2013;121(16):3246-3253.

15. Schindelin J, Arganda-Carreras I, Frise E, et al. Fiji: an open-source platform for biological-image analysis. Nat Methods. 2012;9(7):676-682.

16. Mantel CR, O'Leary HA, Chitteti BR, et al. Enhancing hematopoietic stem cell transplantation efficacy by mitigating oxygen shock. Cell. 2015;161(7):1553-1565.

17. Mohyeldin A, Garzon-Muvdi T, QuinonesHinojosa A. Oxygen in stem cell biology: a critical component of the stem cell niche. Cell Stem Cell. 2010;7(2):150-161.

18. Yeo JH, Cosgriff MP, Fraser ST. Analyzing the formation, morphology, and integrity of erythroblastic islands. Methods Mol Biol. 2018;1698:133-152.

19. Lizarralde Iragorri MA, El Hoss S, Brousse V, et al. A microfluidic approach to study the effect of mechanical stress on erythrocytes in sickle cell disease. Lab Chip. 2018;18 (19):2975-2984.

20. Antoniani C, Meneghini V, Lattanzi A, et al. Induction of fetal hemoglobin synthesis by CRISPR/Cas9-mediated editing of the human beta-globin locus. Blood. 2018;131 (17):1960-1973

21. Zhang Y, Paikari A, Sumazin P, et al. Metformin induces FOXO3-dependent fetal hemoglobin production in human primary erythroid cells. Blood. 2018;132(3):321-333.

22. Kalfa T, McGrath KE. Analysis of erythropoiesis using imaging flow cytometry. Methods Mol Biol. 2018;1698:175-192.

23. Bolte S, Cordelieres FP. A guided tour into subcellular colocalization analysis in light microscopy. J Microsc. 2006;224(Pt 3):213232.

24. Manders EM, Stap J, Brakenhoff GJ, van Driel R, Aten JA. Dynamics of three-dimensional replication patterns during the $\mathrm{S}$ phase, analysed by double labelling of DNA and confocal microscopy. I Cell Sci. 1992;103 ( Pt 3):857-862.

25. Schallmeiner E, Oksanen E, Ericsson O, et al. Sensitive protein detection via triple-binder proximity ligation assays. Nat Methods. 2007;4(2):135-137.

26. Moutouh-de Parseval LA, Verhelle D, Glezer $\mathrm{E}$, et al. Pomalidomide and lenalidomide regulate erythropoiesis and fetal hemoglobin production in human CD34+ cells. J Clin Invest. 2008;118(1):248-258

27. Finch CA, Lee MY, Leonard JM. Continuous $\mathrm{RBC}$ transfusions in a patient with sickle cell disease. Arch Intern Med. 1982;142(2):279 282.

28. Powars DR, Weiss JN, Chan LS, Schroeder WA. Is there a threshold level of fetal hemoglobin that ameliorates morbidity in sickle cell anemia? Blood. 1984;63(4):921-926.

29. Sewchand LS, Johnson CS, Meiselman HJ. The effect of fetal hemoglobin on the sickling dynamics of SS erythrocytes. Blood Cells. 1983;9(1):147-166.

30. Boyer SH, Margolet L, Boyer ML, et al. Inheritance of $\mathrm{F}$ cell frequency in heterocellular hereditary persistence of fetal hemoglobin: an example of allelic exclusion. Am J Hum Genet. 1977;29(3):256-271.

31. Boyer SH, Belding TK, Margolet L, Noyes AN. Fetal hemoglobin restriction to a few erythrocytes ( $\mathrm{F}$ cells) in normal human adults. Science. 1975;188(4186):361-363.

32. Boyer SH, Belding TK, Margolte L, Noyes AN, Burke PJ, Bell WR. Variations in the frequency of fetal hemoglobin-bearing erythrocytes (F-cells) in well adults, pregnant women, and adult leukemics. Johns Hopkins Med J. 1975;137(3):105-115.

33. Wood WG, Stamatoyannopoulos G, Lim G, Nute PE. F-cells in the adult: normal values and levels in individuals with hereditary and acquired elevations of $\mathrm{Hb} \mathrm{F}$. Blood. 1975;46(5):671-682.

34. Stamatoyannopoulos G, Veith R, Galanello $\mathrm{R}$, Papayannopoulou T. Hb F production in stressed erythropoiesis: observations and kinetic models. Ann N Y Acad Sci. 1985;445:188-197.

35. Dover GJ, Boyer SH, Charache S, Heintzelman K. Individual variation in the production and survival of $\mathrm{F}$ cells in sicklecell disease. N Engl J Med. 1978;299(26): 1428-1435.

36. Franco RS, Lohmann J, Silberstein EB, et al. Time-dependent changes in the density and hemoglobin F content of biotin-labeled sick- 
le cells. J Clin Invest. 1998;101(12):27302740.

37. Franco RS, Yasin Z, Palascak MB, Ciraolo P, Joiner CH, Rucknagel DL. The effect of fetal hemoglobin on the survival characteristics of sickle cells. Blood. 2006;108(3):1073-1076.

38. Maier-Redelsperger M, Noguchi CT, de Montalembert M, et al. Variation in fetal hemoglobin parameters and predicted hemoglobin S polymerization in sickle cell children in the first two years of life: Parisian Prospective Study on Sickle Cell Disease. Blood. 1994;84(9):3182-3188.

39. Meiler SE, Wade M, Kutlar F, et al.
Pomalidomide augments fetal hemoglobin production without the myelosuppressive effects of hydroxyurea in transgenic sickle cell mice. Blood. 2011:118(4):1109-1112.

40. Ribeil JA, Zermati Y, Vandekerckhove J, et al. Hsp70 regulates erythropoiesis by preventing caspase-3-mediated cleavage of GATA-1. Nature. 2007;445(7123):102-105.

41. Noguchi CT, Rodgers GP, Schechter AN Intracellular polymerization. Disease severity and therapeutic predictions. Ann N Y Acad Sci. 1989;565:75-82.

42. Paikari A, Sheehan VA. Fetal haemoglobin induction in sickle cell disease. $\mathrm{Br} \mathrm{J}$
Haematol. 2018;180(2):189-200.

43. Howard J, Hemmaway CJ, Telfer P, et al. A phase $1 / 2$ ascending dose study and openlabel extension study of voxelotor in patients with sickle cell disease. Blood. 2019;133(17):1865-1875.

44. Vichinsky E, Hoppe CC, Ataga KI, et al. A phase 3 randomized trial of voxelotor in sickle cell disease. N Engl J Med. 2019;381 (6):509-519.

45. Ribeil JA, Hacein-Bey-Abina S, Payen E, et al. Gene therapy in a patient with sickle cell disease. N Engl J Med. 2017;376(9): 848-855. 